\title{
Aplicación de la legislación ambiental y territorial en municipios capitales: Estudio de caso Villavicencio (Colombia)
}

\section{Applying territorial and environmental legislation in Colombia's capital municipalities: a case study regarding Villavicencio}

\section{Aplicação da legislação ambiental e territorial em municípios capitais: Estudo de caso Villavicencio (Colômbia)}

Martha Lucia Ortiz-Moreno ${ }^{1}$; José Salatiel Rodrigues-Pires ${ }^{1}$

Biologo, PhD, Laboratório de Analise e Planejamento Ambiental-LAPA, Universidade Federal de São Carlos, São Carlos, Brasil. Email: martica4225@yahoo.com

Recibido: : Septiembre 22 de $2013 . \quad$ Aceptado: Junio 27 de 2014.

\section{Resumen}

El municipio es responsable de la definición y ejecución de sus objetivos socioeconómicos en concordancia con la protección del medio ambiente. Para tal fin, debe implementar las políticas ambientales y de ordenamiento territorial que ofrecen herramientas fundamentales para la conservación del medio ambiente y regular el desarrollo de los países. En países megadiversos como Colombia existe un escaso control nacional sobre los alcances y características de los planes de ordenamiento territorial (POT) municipal. El objetivo de esta investigación fue analizar la aplicación de la legislación ambiental y territorial en municipios capitales que están en vías de transformarse en metrópolis, utilizando como estudio de caso el municipio de Villavicencio (Colombia). Se encontró que los municipios incorporan las leyes nacionales en los POT, subordinando la aplicación de la legislación ambiental a los intereses económicos y que faltan mecanismos e instancias supramunicipales de planeación regional. A su vez, los municipios tienen limitaciones económicas, administrativas y técnicas para establecer mecanismos y la ejecución de sus POT.

Palabras clave: Colombia, políticas ambientales, ordenamiento territorial, Villavicencio.

\section{Abstract}

A municipality is responsible for defining and executing its own socioeconomic objectives in line with environmental protection; this means that environmental and zoning policy must be implemented thereby offering fundamental tools for environmental conservation and regulating countries' development. There is little national control over the scope and characteristics of municipal territorial ordering plans (TOP)/land use in mega-diverse countries like Colombia. This research was aimed analysing the application of environmental and territorial legislation in 
capital municipalities which are becoming metropolises, using the town of Villavicencio (Colombia) as case study. It was found that the municipalities incorporated national laws in the TOP, subordinating the application of environmental legislation to economic interests, and that supra-municipal/regional mechanisms and planning bodies are lacking. In turn, the municipalities suffer economic, administrative and technical limitations for establishing mechanisms and executing their TOP.

Key words: Colombia, environmental policy, territorial ordering/zoning/land use, Villavicencio.

\section{Resumo}

O município é responsável pela definição e execução de seus objetivos socioeconômicos, de acordo com a proteção ambiental, para isso, deve programar as políticas ambientais e territoriais que oferecem ferramentas básicas para a conservação do ambiente e para regular o desenvolvimento dos países. Em países megadiversos, como a Colômbia há pouco controle nacional sobre o alcance e as características dos planos diretores municipais (POT). O objetivo desta pesquisa foi analisar a aplicação da legislação ambiental e territorial em municípios capitais que estão em processo de se tornar metrópoles, utilizando como estudo de caso o município de Villavicencio (Colômbia). Encontro-se que os municípios incorporam as leis nacionais nos POT, privilegiando os interesses econômicos à aplicação da legislação ambiental e faltam mecanismos além de instancias supramunicipais de planejamento regional. Por sua vez, os municípios têm limitações econômicas, administrativas e técnicas para estabelecer mecanismos e a execução de seus POT.

Palavras chave: Colômbia, políticas ambientais, ordenamento territorial, Villavicencio.

\section{Introducción}

Colombia es un país megadiverso, extremadamente rico en especies y ecosistemas, debido a sus particulares condiciones geográficas, ya que es el único país Sudamericano con costas en el Océano Pacífico y el Mar Caribe (Mittermeier, et al., 1997). Colombia posee la parte norte de las montañas de los Andes y más del $50 \%$ de su territorio se encuentra en las cuencas hidrográficas del Orinoco y Amazonas. El territorio continental de Colombia tiene aproximadamente 1,1 millones de $\mathrm{Km}^{2}$, y comprende cinco regiones naturales: Andina, Caribe, Pacífica, Amazónica, y Orinoquía (sabanas influenciadas por la cuenca del río Orinoco) (Etter, et al., 2008). En este territorio se presentan formaciones vegetales tales como los bosques secos y andinos (9\%), sabanas $(13.9 \%)$ y selvas húmedas (52.2\%) (Armenteras, et al., 2009; ArmenterasPascual, et al., 2011).

Sin embargo esta gran diversidad de paisaje y biológica se encuentra amenazada, principalmente por la acelerada deforestación y los cambios en la cobertura vegetal inducidos por el ser humano. En Colombia, desde 1500 hasta el año 2000, 80\% del área fue deforestada o se ha cambiado su cobertura vegetal, para implantar principalmente áreas de pastaje para la cría extensiva de ganado. Las selvas húmedas de tierras bajas y los bosques secos y andinos son los más afectados por este fenómeno (Mendoza y Etter, 2002;

Aplicación de la legislación ambiental y territorial en municipios capitales: Estudio de caso Villavicencio (Colombia) 
Etter, et al., 2008). En principio, la deforestación responde a intereses económicos. Inicialmente, se concentró en la región Andina y Caribe del país, para desplazarse en lo reciente a las regiones Amazónica y Pacífica, con el avance de la frontera agropecuaria y los cambios en la distribución de la población en el territorio (Etter, et al., 2008; Etter, et al., 2006; Prieto y Luengas, 2011). La deforestación tiene un vertiginoso avance en Colombia que alcanza 100.000ha/año y sus efectos en la diversidad biológica son incalculables (IDEAM, 2004; Etter, et al., 2006; Etter, et al., 2008).

Colombia es un país prolífico en legislación para la mayoría de sus aspectos y ha estado acompañando la preocupación mundial por el desarrollo sustentable (Massiris, 2000; 2002; García, 2003; Baker, 2006; Ucros, 2008; Gutiérrez, 2010; Rauschmayer, et al., 2012). Con el objetivo de regular el uso de los recursos naturales, la legislación ambiental nacional surgió en los años 50's. Las áreas de protección ambiental fueron creadas en los años 70's y 90's para conservar recursos estratégicos, y en 1974, con el decreto 2811, se crea el Código Nacional de Recursos Naturales Renovables y de Protección del Medio Ambiente, que sienta las bases para el ordenamiento ambiental del territorio. Establece un gran número de categorías espaciales, agrupadas en el Sistema de Parques Nacionales Naturales, que incluye las siguientes categorías: parque nacional, reserva natural, área natural única, santuario de fauna, vía parque (Massiris, 2000; Ucros, 2008).

En 1993, se crea el Ministerio de Medio Ambiente y el Sistema Nacional Ambiental con la Ley 99, que retoma y desarrolla varias categorías de ordenamiento ambiental del Código de Recursos Naturales Renovables e introduce elementos importantes relativos a la dimensión ambiental del ordenamiento territorial: zonificación del uso del territorio para su apropiado ordenamiento ambiental; regulaciones nacionales sobre usos del suelo en lo concerniente a sus aspectos ambientales; pautas ambientales para el ordenamiento y manejo de cuencas hidrográficas y demás áreas de manejo especial; regulación ambiental de asentamientos humanos y actividades económicas; reglamentación de usos de áreas de parques nacionales naturales (Massiris, 2000; Ucros, 2008).

A la par de las políticas ambientales, Colombia ha construido una legislación que regula las modificaciones del uso del suelo y el desarrollo, para obtener un territorio ordenado y armónico. La legislación de ordenamiento territorial (OT) surge a finales de la década de los 40's, específicamente orientada a los grandes centros poblados. Después de la Conferencia Hábitat (1976), se integra el componente ambiental al OT y la armonía en el desarrollo zonal (Massiris, 2000; 2002). En general, existe en la legislación colombiana una fuerte asociación entre el OT y las políticas ambientales, siendo que estas últimas generalmente están subordinadas a las prioridades gubernamentales de desarrollo del territorio (Carrión, 2008; Prieto y Luengas, 2011).

En cuanto al ordenamiento rural, la primera reforma agraria se establece en 1961 con la Ley 135, que intenta modificar la histórica concentración de tierras rurales e introduce las zonas de colonización (Massiris, 2000; Etter, et al., 2006; 2008). Esta iniciativa es retomada en 1994 con la Ley 160, que establece categorías de ordenamiento rural relacionadas con las áreas de baldíos nacionales, zonas de colonización y zonas de reserva campesina. Sin embargo los conflictos por la tenencia de la tierra en el país no han sido resueltos hasta la actualidad (Rausch, 2007; Etter, et al., 2008; Rodríguez, et al., 2011).

Con la Constitución Política de 1991, se establece un nuevo orden territorial para 
Colombia, basado en cinco componentes básicos: a) Ordenamiento PolíticoAdministrativo, presente en los siguientes artículos: Art. 288, Distribución de competencias entre la nación y entidades territoriales; Art. 297, Formación de nuevos departamentos; Art. 307, Conversión de regiones en entidades territoriales; Art. 319: Régimen de áreas metropolitanas; Art. 329: Conformación de entidades territoriales. b) Ordenamiento del Desarrollo Municipal: Art. 311. c) Ordenamiento Ambiental: Art. 58: Función social y ecológica de la propiedad; Art. 80: Aprovechamiento sostenible de recursos naturales renovables; Art. 313: Reglamentación de usos del suelo; Art. 334: Intervención del Estado sobre la economía para la preservación de un ambiente sano. d) Desarrollo Regional Armónico: Art. 334. e) Ordenamiento del Desarrollo Social Urbano y Rural: Art. 64 y 65: Ordenamiento del desarrollo campesino; Art. 334: Distribución equitativa de oportunidades y beneficios del desarrollo; Art. 334: Acceso de personas con bajos ingresos a bienes y servicios básicos (Massiris, 2000; Ucros, 2008).

La unidad administrativa del planeamiento es el municipio y es responsable de la definición y ejecución de sus objetivos socioeconómicos en concordancia con la protección del medio ambiente. A partir de 1994 se establecen las bases del OT municipal. Con la Ley 152 (Ley Orgánica del Plan de Desarrollo) se establece la obligatoriedad de los municipios para realizar planes de ordenamiento territorialPOT (Art. 41), complementarios al plan de desarrollo. La Ley 388 de 1997 establece el marco conceptual e instrumental para formular y ejecutar planes municipales y distritales de ordenamiento territorial. En 1999, la Ley 507 modifica la Ley 388, en términos de ampliar el plazo máximo para la formulación y adopción de los planes y esquemas de OT, hasta el 31 de diciembre de 1999 (Massiris, 2000; Ucros, 2008). Es a partir del año 2000, que muchos POT y Esquemas de
OT nacionales entran en vigencia y actualmente se encuentran en proceso de revisión y ajuste.

Con la creciente economía y la creciente concentración de la población urbana en los municipios capitales, las ciudades intermedias en Colombia están en vías de convertirse en metrópolis. Es por ello que los POT de estos municipios deben desarrollar de manera armónica los aspectos socio-económicos y ambientales para garantizar un desarrollo sustentable (García, 2003; Baker, 2006; Rauschmayer, et al., 2012). El objetivo de este trabajo fue analizar la aplicación de la legislación ambiental y de OT a nivel municipal en municipios capitales y se propone usar como estudio de caso a Villavicencio (Departamento del Meta, Colombia). Este municipio se caracteriza por una alta actividad económica determinada por ser la capital del Departamento del Meta, centro comercial y de servicios para toda la Orinoquía, además de actuar como receptor de población desplazada por el conflicto interno oriunda de diferentes regiones del país (Rausch, 2007; Alcaldía de Villavicencio, 2009).

\section{Materiales y métodos}

Para realizar el análisis de aplicación de la legislación nacional y municipal sobre medio ambiente y ordenamiento territorial, se realizó un levantamiento bibliográfico con los recursos disponibles en internet en portales oficiales, artículos e información suministrada por la actual Secretaria de Planeación del municipio de Villavicencio. Con base en los documentos recopilados se realizó un análisis crítico de la información (Tabla 1).

Los municipios capitales colombianos con ciudades intermedias cuyos POT fueron analizados en lo que refiere a la aplicación de la legislación ambiental y de OT, se

Aplicación de la legislación ambiental y territorial en municipios capitales: Estudio de caso Villavicencio (Colombia) 
seleccionaron según su prospectiva oficial de número de habitantes para el año 2013 (DANE, 2013): Santa Marta (Departamento Magdalena, 468.962hab), Villavicencio (Departamento Meta, 463.093hab), Manizales (Departamento Caldas, 393.189hab), Buenaventura (Departamento Valle del Cauca, 384.402hab), Neiva (Departamento Huila, 337.943hab); Armenia (Departamento Quindío, 293.614hab).

Tabla 1. Recursos bibliográficos consultados para el análisis de la aplicación legislativa en los POT

\begin{tabular}{|c|c|}
\hline Tema & Fuente \\
\hline $\begin{array}{l}\text { Leyes nacionales ambientales } \\
\text { y de OT en su contexto }\end{array}$ & $\begin{array}{l}\text { Massiris, 2000; 2002; Ucros, } 2008 \\
\text { Rodríguez et al., 2011; Escobar, } 2013\end{array}$ \\
\hline $\begin{array}{l}\text { Referentes legislativos } \\
\text { ambientales y de OT para } \\
\text { los POT de municipios } \\
\text { capitales }\end{array}$ & $\begin{array}{l}\text { Alcaldía de Armenia, } 2009 \\
\text { Alcaldía de Buenaventura, } 2001 \\
\text { Curaduría de Manizales, 2001; 2003; } 2007 \\
\text { Alcaldía de Neiva, 2000; 2009; } 2010 \\
\text { Alcaldía de Santa Marta, 2000; } 2012 \\
\text { Alcaldía de Villavicencio. 2000; } 2011\end{array}$ \\
\hline $\begin{array}{l}\text { Análisis del componente } \\
\text { general del POT de } \\
\text { Villavicencio }\end{array}$ & Alcaldía de Villavicencio. 2000; 2009; 2011; 2012. \\
\hline Mapas temáticos & Alcaldía de Villavicencio. 2009; IGAC, 2003. \\
\hline
\end{tabular}

Los mapas temáticos modificados correspondientes a la ubicación, unidades fisiográficas (denominadas paisajes en el POT) y cuencas hidrográficas del municipio fueron elaborados a partir de la digitalización de los mapas oficiales disponibles en el portal de la Alcaldía de Villavicencio, en ambiente SIG (Sistema de Información Geográfica), utilizando el software Mapinfo 11.

\section{Área de estudio}

Villavicencio es un municipio localizado en las coordenadas $04^{\circ} 09^{\prime} \mathrm{N} 73^{\circ} 38^{\prime} \mathrm{O}$, en la interface entre la cordillera oriental y las sabanas colombianas; surgió como un asentamiento de cruce de caminos en las rutas comerciales de la Orinoquía y se convirtió en municipio por su posición estratégica, que permite la conexión comercial entre la Orinoquía y Bogotá, la capital del país (Figura 1). Este municipio es la capital del Departamento del Meta y ha venido transformándose en una metrópoli, gracias a múltiples flujos de migración (ciudad boom) asociados a diferentes factores como actividades económicas (agropecuarias y mineras), políticas gubernamentales y 
el conflicto interno nacional. Los migrantes que han colonizado Villavicencio son oriundos, principalmente, de la región Andina y del interior de la Orinoquia con culturas y prácticas agropecuarias diferentes (Rausch, 2007).

La historia de Villavicencio ha sido marcada desde su fundación, en 1840, por su difícil acceso a Bogotá. La vía que comunica a las dos ciudades había sido una gran limitante para el comercio regional, y no tendría mejoras significativas hasta finales de los 90's. El crecimiento del municipio estuvo influenciado por el liderazgo de la iglesia católica (especialmente jesuitas y montfortianos) que trabajó en pro del bienestar social y por la violencia partidista que sumió la Orinoquía en el abandono por ser un bastión liberal que no tuvo una recuperación socioeconómica hasta el gobierno de Alfonso López Pumarejo (liberal, 1934-1938) (Rausch, 2007).

La explotación petrolera se inició en 1940`s con la Tropical Oil Company y luego por ECOPETROL (Empresa nacional de carácter mixto) (1970`s), que trabaja con un sistema de concesiones a compañías como Continental, International Petroleum Colombian, British Petroleum y Superior Oil. En la actualidad, este sistema se mantiene en el territorio del Departamento del Meta (Rausch, 2007).

A partir de 1977 inició la influencia del narcotráfico en la Orinoquia con el cultivo de marihuana. Posteriormente, los carteles de la droga estimularon el cultivo de la coca (19801986). El flujo de dinero generado por el narcotráfico estimuló la migración, la urbanización y crecimiento del sector comercial y de servicios, ya que generó un gran aumento del poder adquisitivo de la población. Sin embargo, el narcotráfico trajo consigo un recrudecimiento de la violencia al ofrecer recursos ilimitados para sustentar grupos al margen de la ley, como la guerrilla y paramilitares, los cuales incrementaron sus operaciones. Aquelló generó grandes oleadas de desplazados en el interior de la Orinoquia, que sumados a los migrantes venidos de otros Departamentos, hicieron de Villavicencio un centro de recepción de población desplazada. Por otra parte, la mejora en el acceso a Villavicencio y de su infraestructura hotelera incentivó el desarrollo del turismo, que actualmente es uno de los principales renglones económicos del municipio (Rausch, 2007; Prieto y Luengas, 2011).

Villavicencio posee tres unidades fisiográficas (denominadas paisajes según el POT): Cordillera: Corresponde a la zona montañosa constituida por las estribaciones de la vertiente oriental de la cordillera oriental, hasta alcanzar alturas de subpáramo y páramo, oscila entre los 1000msnm y los 3700msnm; Piedemonte: Situado antes del encumbramiento de la Cordillera Oriental, conforma una faja de terreno con pendiente moderada a alta, cuya altura sobre el nivel del mar oscila entre los 400 y los $1000 \mathrm{msnm}$; alcanza zonas medias de cuencas hídricas, abanicos aluviales y está conformada por materiales coluviales provenientes de las partes altas de la cordillera. Llanura: En la que se encuentra la planicie aluvial de desborde, el plano aluvial marginal, terrazas aluviales, los valles y los abanicos que conforman parte de las cuencas media y baja de los ríos Ocoa, Guatiquía, Negro y Guayuriba. (Alcaldía de Villavicencio, 2000) (Figura 2).

El municipio tiene un clima húmedo tropical (Temperatura media de $27^{\circ} \mathrm{C}$ y $78 \%$ de humedad relativa), con una altura media de 467 msnm y se encuentra delimitado por los ríos Guatiquía y Guayuriba (Figura 2) (Alcaldía de Villavicencio, 2012).

Aplicación de la legislación ambiental y territorial en municipios capitales: Estudio de caso Villavicencio (Colombia) 

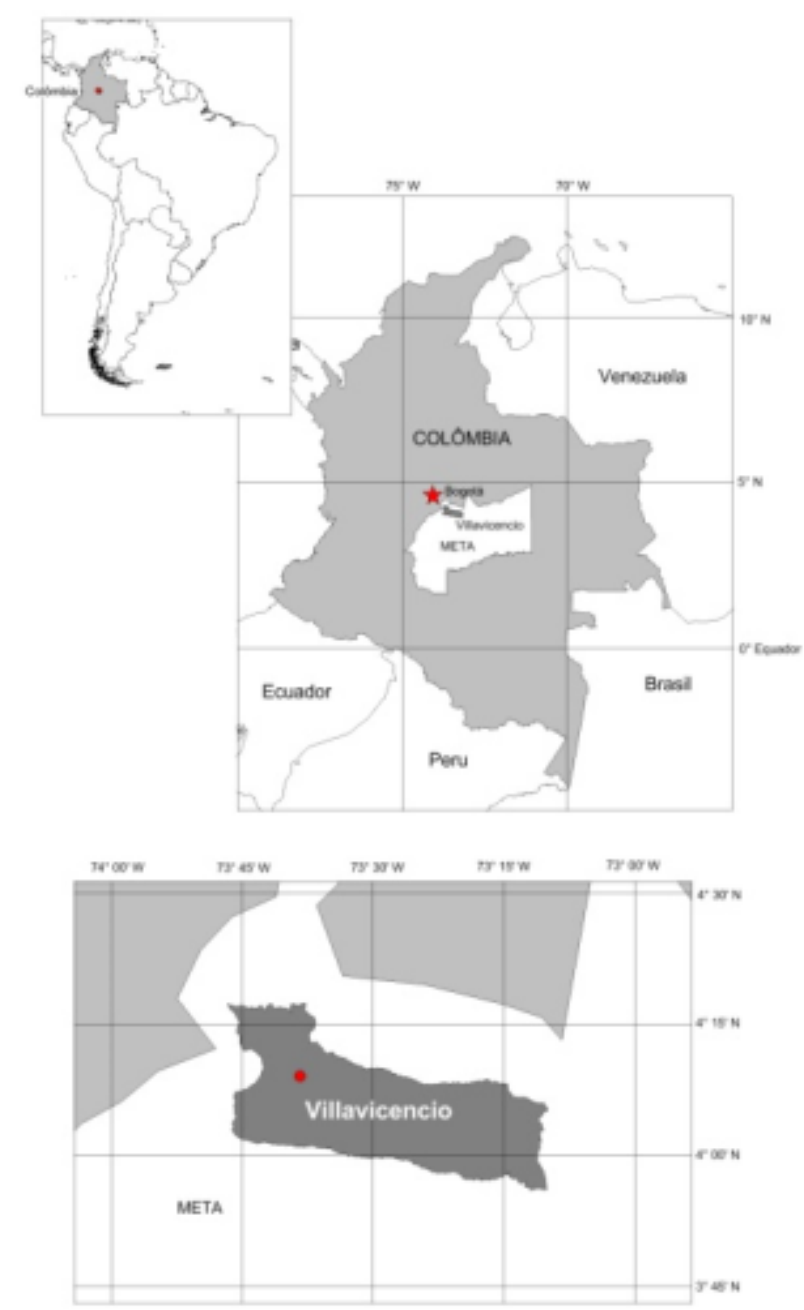

Modicado lonc

Org Ortie Moreno (2103)

Figura 1. Mapa de ubicación geográfica de Villavicencio (Meta, Colombia) (IGAC, 2003)

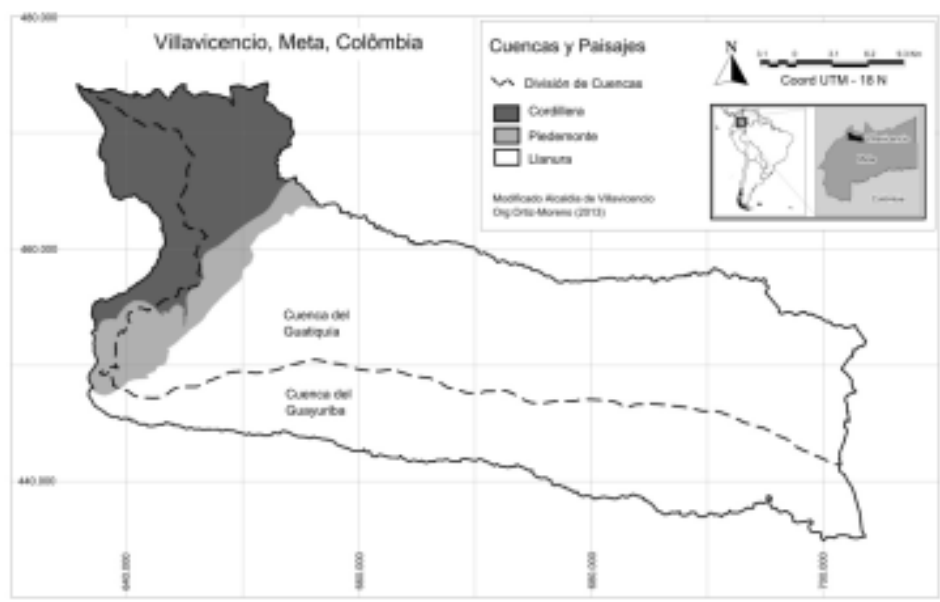

Figura 2. Mapa de cuencas y paisajes del municipio de Villavicencio (Alcaldía de Villavicencio, 2009). 
Villavicencio posee, aproximadamente, 460.000 habitantes distribuidos en un total de 133.085ha, 5.208ha de área urbana y 127.877ha de área rural, organizadas en 7 corregimientos (Alcaldía de Villavicencio, 2009; DANE, 2013).

El área urbana de Villavicencio se divide en 8 comunas con 320 barrios (101 sin reconocimiento por los órganos municipales) y 446 asentamientos (urbanizaciones diferentes a barrios), de los cuales 245 no tienen legalización urbanística. En el Plan de Ordenamiento Territorial (POT) se consideran comunas a las unidades con, al menos, 50.000 habitantes; y los barrios poseen al menos 5.000 habitantes. Entre 1973 y el presente, el área urbana de Villavicencio se ha expandido más rápido que cualquier otra ciudad en Colombia. Villavicencio tiene un alto crecimiento demográfico con un débil desarrollo industrial; su economía desbalanceada produce marginalización, sobreurbanización, excesivo desempleo e insuficiente oferta de agua, electricidad y alcantarillado para satisfacer las necesidades de la creciente población. El área rural del municipio se divide en 4 zonas geoeconómicas que poseen 11 centros poblados y 29 asentamientos. Sus principales productos agropecuarios son el ganado, el arroz, la palma africana, el plátano, los cítricos, la soya, la yuca, el maíz, el cacao, el sorgo y la papaya. A su vez, posee explotaciones activas de petróleo, gas, arena y grava de rio (Alcaldía de Villavicencio, 2000;2009; Rausch, 2007).

El municipio se desarrolló siguiendo los ejes definidos por las vías principales que lo atraviesan. Eso genera una fuerte centralización del área urbana con escaso espacio público, expansión urbana en las áreas montañosas y de piedemonte, paralelas a la antigua y actual vía BogotáVillavicencio e invasión de las márgenes de los ríos y de los derechos de vía (100m de las vías nacionales y departamentales) (Rausch, 2007).

\section{Resultados}

\section{Referentes legislativos ambientales y de OT para los POT}

Hay una extensa legislación ambiental y de OT citadas en los POT de los municipios capitales seleccionados en los que la Constitución Política de 1991 es fundamental. En materia ambiental, el principal referente fue la Ley 99 de 1993, si bien aún se cita el Decreto 2811 de 1974 (Código de Nacional de Recursos Naturales Renovables). La Ley 388 de 1997, que da las bases para la construcción de los POT, es fundamental para el OT. Las leyes 9/89, 136/94 y 160/94, junto con los Decretos 879/98 y 1504/98, también son importantes (Anexo, Tabla 2).

\section{Estudio de caso: POT Villavicencio}

En el año 2000, mediante Decreto 353, el municipio de Villavicencio adoptó su POT, el cual fue modificado con el Acuerdo 021 de 2002, definiendo un conjunto de normas que orientan el uso, la ocupación y el aprovechamiento del suelo del territorio municipal (Alcaldía de Villavicencio, 2000). EI POT fue construido bajo los parámetros establecidos en la Constitución de 1991 y la Ley 388 de 1997 (Alcaldía de Villavicencio, 2000) (Anexo, Tabla 2). En concordancia con el Artículo 28 de la Ley 388 de 1997, Artículo 1 de la Ley 507 de 1999, los artículos 7 y 8 del Decreto 879 de 1998 y la Ley 546 de 1999 y demás normas que lo adicionen modifiquen o complementen, el POT se adopta para una vigencia correspondiente a tres (3) períodos constitucionales de la Administración Municipal. Esto quiere decir que son 12 años contados a partir de la fecha de su puesta en vigencia; por eso está en proceso de revisión y actualización desde el 2011, y la propuesta para su modificación es el proyecto de 
Acuerdo 018 de 2011 (Alcaldía de Villavicencio, 2000; 2011).

\section{Análisis del componente general del POT de Villavicencio}

La Ley 388 de 1997, establece que el POT municipal se debe dividir en tres componentes: General, Urbano y Rural. Dentro del componente general el municipio debe definir los objetivos y estrategias territoriales de largo y mediano plazo, realizando la 1.1 Identificación y localización de las acciones sobre el territorio que posibiliten organizarlo y adecuarlo para el aprovechamiento de sus ventajas comparativas y su mayor competitividad; 1.2 Definición de las acciones territoriales estratégicas necesarias para garantizar la consecución de los objetivos de desarrollo económico y social del municipio o distrito; 1.3 Adopción de las políticas de largo plazo para la ocupación, aprovechamiento y manejo del suelo y del conjunto de los recursos naturales.

\section{Objetivos y Estrategias del POT de Villavicencio}

La tabla 3 presenta los objetivos de desarrollo y las estrategias propuestas para alcanzarlos (Alcaldía de Villavicencio, 2000). Estos componentes no han sido modificados en el Proyecto de Acuerdo 018 de 2011. Los primeros cuatro objetivos están relacionados con el numeral 1.1 de la Ley 388/97. En tanto que las estrategias son producto de la aplicación del numeral 1.2.

\section{Discusión}

El territorio es el escenario físico y ambiental en donde se dinamiza y convergen los procesos de desarrollo. Por lo tanto, constituye un elemento integrador entre actividades y relaciones sociales, económicas y culturales del hombre. EI OT y ambiental es una construcción social procesual autoorganizada que presupone una orden integral del territorio, que se debe ver reflejada en los POT (Prieto y Luengas, 2011).

Los POT pretenden alcanzar niveles óptimos de gobernabilidad, productividad y habitabilidad; han de tener en cuenta la conectividad, competitividad y el mercado, los cuales son componentes indispensables, comunes y transversales al desarrollo. Estos factores impulsan los procesos de ordenamiento territorial (Yory, 2007; Espinosa, 2008).

Se justifica presentar consideraciones sobre la normatividad que define parámetros e instrumentos para la organización del espacio, así como evidenciar la necesidad de tener en cuenta el aprovechamiento y la protección de los recursos naturales de Colombia, bajo estas normas, y retribuir al OT como herramienta clave para la gestión ambiental. Lo anterior, debido a los múltiples inconvenientes que presentan los municipios de Colombia, para establecer políticas territoriales en temas de ordenamiento y de medio ambiente articuladas entre sí; además de las dificultades para la construcción de directrices territoriales con su entorno geográfico (Prieto y Luengas, 2011).

\section{Referentes legislativos ambientales y de OT para los POT}

El principal referente en materia ambiental para los POT analizados es la Ley 99 de 1993 (Anexo, Tabla 2). Esta ley incluye los postulados y resultados de la Cumbre de la Tierra (Conferencia de Rio 1992) y desarrolla los aspectos ambientales de la Constitución Política de 1991. Fue elaborada bajo el mandato del Presidente Cesar Gaviria, periodo en el cuál se inició la Apertura Económica que afecto profundamente la economía del país, al eliminar las barreras arancelarias a los 
productos importados, en una época de guerra frontal contra la mafia y la guerrilla (Rodríguez, et al., 2011; Escobar, 2013).

La ley consta de 118 artículos y 17 títulos e incorpora el concepto de desarrollo sostenible. Dentro de sus principios generales incluye la protección de la biodiversidad, el derecho a una vida saludable en armonía con la naturaleza que debe ser tenida en cuenta en las políticas de población, protección de zonas de recarga de acuíferos y nacimientos de agua, así como la prioridad del consumo humano sobre cualquier otro uso de los recursos hídricos. Señala la protección del paisaje como prioritaria por ser patrimonio común (Escobar, 2013).

La Ley 99 regula como principio la incorporación de costos ambientales y el uso de instrumentos económicos para fines ambientales. Así mismo, consagra que la acción para la protección y recuperación del ambiente es tarea conjunta entre los particulares y el estado. Define los estudios de impacto ambiental como un instrumento básico para la toma de las decisiones respecto a la construcción de obras y actividades que afecten significativamente el ambiente y realiza el desarrollo normativo de las licencias ambientales. Retoma las tasas retributivas y compensatorias definidas por el Decreto 2811 de 1974. Señala que el manejo ambiental del país, conforme a la Constitución Política, debe ser descentralizado, democrático y participativo, y que las instituciones ambientales del Estado se estructuran teniendo como base criterios de manejo integral del medio ambiente y su interrelación con los procesos de planificación económica, social y física. Definió el Sistema Nacional Ambiental (SINA) para dar cumplimiento a los objetivos ambientales planteados en la Constitución Política de 1991 y definió el esquema institucional encargado del medio ambiente en el país (Escobar, 2013).
En cuanto al OT, la base legislativa para la construcción de los POT es la Ley 388 de 1997 (Anexo, Tabla 2). Esta ley fue promulgada bajo el mandato del Presidente Ernesto Samper Pizano, cuyo gobierno estuvo marcado por el escándalo de dineros del narcotráfico que financiaron la campaña presidencial y el recrudecimiento de la violencia asociado al fortalecimiento del tráfico de drogas (Rodríguez et al., 2011).

La Ley 388 pretende armonizar y actualizar las disposiciones contenidas en la Ley 9 de 1989 con las nuevas normas establecidas en la Constitución Política, la Ley Orgánica del Plan de Desarrollo, la Ley Orgánica de Áreas Metropolitanas y la Ley 99/93. Estableciendo mecanismos que permitan al municipio promover el ordenamiento de su territorio, el uso equitativo y racional del suelo; la preservación y defensa del patrimonio ecológico y cultural, junto con la prevención de desastres en asentamientos de alto riesgo (Prieto y Luengas, 2011).

La Ley 388 establece el marco conceptual e instrumental para formular y ejecutar planes municipales y distritales de ordenamiento territorial. Concibe el OT como un conjunto de acciones político-administrativas y de planificación física concertadas, emprendidas por los municipios o distritos y áreas metropolitanas en orden a disponer de instrumentos eficientes para orientar el desarrollo del territorio y regular la utilización, transformación y ocupación del espacio, de acuerdo con las estrategias de desarrollo socioeconómico y en armonía con el medio ambiente y las tradiciones históricas y culturales (Art. 5). El Objeto de esta política es el de complementar la planificación económica y social, introduciendo la dimensión territorial en la búsqueda de racionalizar las actuaciones sobre el territorio y orientar su desarrollo y aprovechamiento sostenible a partir de estrategias territoriales de uso, ocupación y manejo del suelo, en función

Aplicación de la legislación ambiental y territorial en municipios capitales: Estudio de caso Villavicencio (Colombia) 
de objetivos económicos, sociales y ambientales y la articulación de las actuaciones sectoriales que afecten la estructura del territorio (Art. 6) (Massiris, 2000).

Existe un sesgo de la Ley 388 hacia los aspectos urbanos y del uso del suelo, dejando de lado el ordenamiento rural. Como consecuencia de lo anterior, ofrece las mismas reglas de juego para municipios con tipologías territoriales de naturaleza distinta. Todo esto implica una concepción incompleta del ordenamiento espacial. Este argumento sin duda alude a un problema en la forma como están concebidos los POT. Sin embargo, es necesario decir que esa preferencia de la ley ocurre con amplio desarrollo en los POT que operan en municipios grandes, cuya realidad es sustancialmente urbana y concentran la mayor cantidad de población del país (Gutiérrez, 2010; Gomez et al 2011).

Debido al ámbito municipal de la Ley 388, los POT no tienen herramientas ni recursos suficientes para garantizar la cooperación entre las jurisdicciones territoriales de los municipios vecinos, lo cual limita la ejecución de los objetivos de desarrollo plateados en el componente general de los POT (Gómez et al 2007; Gutiérrez, 2010) (Anexo, tabla 3). Esto se debe a las condiciones de total aislamiento institucional respecto a sus municipios vecinos. Sobre este particular, el establecimiento de una nueva institucionalidad intermunicipal, debería ser uno de los retos que se impusieran los POT, con el objetivo de construir una administración regional fuerte (Yory, 2007).

Desde esta perspectiva, la visión a largo plazo propia de los POT, debe incluir la adopción de un plan capaz de dar respuesta a las contingencias por la vía de la incorporación de la incertidumbre y vulnerabilidad, partiendo de una clara respuesta a la pobreza y generación de empleo. Aunque los POT se orientan a la generación de riqueza, su modelo de distribución de cargas y beneficios debe contemplar una base sustentable que propicie el incremento equitativo de la base productiva en el marco de la generación de un equilibrio tanto económico y ambiental, como social. Ya que el aumento del atractivo de las ciudades y municipios, fortalece los procesos inmigratorios, fenómeno que es observado en Villavicencio (Alcaldía de Villavicencio, 2009). Si lo que se quiere es propiciar un desarrollo territorial equilibrado para la región, el atractivo debe ser desconcentrado por la vía del POT; con el fin, no sólo de impedir la saturación de la "capacidad de carga" del municipio, sino de propiciar nuevas oportunidades para la población por la descentralización de la oferta de capacitación, servicios y empleo. Eso alienta el aumento en la diversidad de la producción (Yory, 2007).

La creación de instancias supramunicipales que funcionen como unidades técnicas de planeación y apoyo al desarrollo territorial integrado cumpliría un papel fundamental en el punto anterior. Dichas instancias, promovidas directamente desde el orden departamental, permitirán la satisfacción de los objetivos tanto del plan de desarrollo departamental, como del propio POT de los municipios (Yory, 2007). En este sentido la Ley Orgánica de Ordenamiento Territorial (Ley 1454 de 2011) podría fortalecer este proceso de cooperación regional (Observatorio legislativo, 2011). Sin embargo, esta ley aún no ha sido incorporada en los POT analizados.

Para las alcaldías, la jerarquía de aplicación de los referentes legislativos ambientales y de OT es la siguiente: 1. Constitución Política. 2. Ley 388 de 1997 y sus decretos reglamentarios. 3 . Ley 99 de 1993. 4. Acuerdo del POT (Alcaldía de Neiva, 2009). Eso muestra que la aplicación de la legislación ambiental está subordinada a las pautas de desarrollo económico y territorial. 
Por su parte, Gómez et al. (2007) y Gutiérrez (2010) afirman que la reglamentación de las políticas ambientales en el POT es insuficiente; que tales políticas son tratadas como base normativa, pero sin que exista una coherencia suficiente que permita armonizar esas políticas ambientales con los POT. Esto representa un problema puesto que, además del incumplimiento de uno de los propósitos fundamentales de la ley, que consiste en armonizar la legislación existente, se estaría entorpeciendo con ello la coherencia de las políticas territoriales, y por tanto, impidiendo el desarrollo territorial. Aunque ocurra una creación formal de los POT, en ocasiones los municipios no poseen ni los recursos, ni la capacidad administrativa para implementarlos. Entonces los planes quedan como una carta de buenas intenciones. Este escenario se agrava cuando los POT de los municipios presentan debilidades técnicas.

\section{Estudio de caso: POT Villavicencio}

\section{Objetivos y Estrategias del POT de Villavicencio}

Debido a que algunos de los objetivos que plantea el POT no son de exclusiva competencia del municipio, no se han logrado cumplir. Por ejemplo, los ítems 1 y 3 que dependen de las intenciones gubernamentales nacionales y regionales. Por tanto resultan totalmente ineficaces, ya que históricamente el municipio ha recibido un escaso apoyo gubernamental debido a su pobre representación política y a que el mismo POT no propone mecanismos específicos para alcanzarlos (Alcaldía de Villavicencio, 2000; Rausch, 2007). El objetivo 5 se sugiere que sea eliminado ya que es una meta de desarrollo administrativo y no municipal (Tabla 3).

En cuanto a las estrategias municipales, cabe resaltar que el municipio tiene un desarrollo urbano desordenado, a pesar de sus 174 años de antigüedad. Por ello, las estrategias de los objetivos 1 y 4 hacen referencia a este tema y el ordenamiento del suelo rural no es considerado todavía como un aspecto a tener en cuenta (Tabla 3). Las estrategias referentes al manejo del agua responden a una paradójica e histórica falta de este servicio público en el municipio, que aún en la actualidad tiene déficit de abastecimiento y no existe alcantarillado pluvial. Con los nueve meses de lluvias por año que se presentan en Villavicencio, hay frecuentes inundaciones en las áreas planas y deslizamientos en la cordillera y piedemonte (Rausch, 2007; Alcaldía de Villavicencio, 2009). Este fenómeno no es aislado, municipios capitales con alta actividad económica como Buenaventura (Puerto), poseen importantes deficiencias en sus coberturas de servicios públicos (Alcaldía de Buenaventura, 2013), debidas al desarrollo desarmónico de las regiones del país (Etter et al., 2006; 2008; Rodríguez, et al., 2011).

En la actualidad el proyecto de Acuerdo 018 de 2011 no modifica los objetivos y estrategias planteadas por el POT de Villavicencio del año 2000 y la falta de recursos económicos e instrumentos institucionales han hecho que después de 14 años continúen sin cumplirse. Esto invita a la Administración municipal a replantear el componente general del POT, ir más allá de las definiciones establecidas por la Ley 388/1997 y formular un nuevo norte municipal para los próximos 12 años, que tenga en cuenta la historia y las necesidades crecientes del municipio.

\section{Conclusiones}

1. Los principales referentes legislativos en materia ambiental y de OT para los POT analizados fueron la Ley 99 de 1993 y la Ley 388 de 1997, mientras que el referente general fue la Constitución Política. La legislación ambiental se encontró subordinada a la territorial, privilegiando el desarrollo económico de los municipios 
frente a la conservación ambiental. Ello se ve reflejado en la estructura de los POT, donde el componente ambiental tenía un carácter complementario y no decisorio.

2. Los POT municipales actúan de forma aislada y la Ley Orgánica de Ordenamiento Territorial (Ley 1454 de 2011) no ha logrado establecer una coordinación efectiva entre las instancias supramunicipales para lograr la cooperación intermunicipal y la planeación regional, especialmente desde el punto de vista de las regiones naturales del país.

3. La falta de herramientas y recursos en el desarrollo desarmónico regional de Colombia y la representación política desigual, son obstáculos que deben ser superados para alcanzar un verdadero ordenamiento territorial ambiental encaminado al desarrollo sustentable, tal y como lo promulga la Constitución Política Nacional. Esto se puede evidenciar en el POT de Villavicencio, con objetivos y estrategias ambiciosas y bien intencionados, pero la falta de apoyo gubernamental nacional y regional limitan sus alcances. A su vez, en el POT no se plantean mecanismos para ejecutar sus propuestas y el ordenamiento rural no es claro, lo cual hace que hasta el momento, su ejecución sea parcial.

\section{Agradecimientos}

A la Alcaldía de Villavicencio, en especial a la Secretaría de Planeación, por la información suministrada. Al soporte financiero dado por el Departamento Administrativo de Ciencia y Tecnología de Colombia-COLCIENCIAS, a través de su convocatoria 497 Jóvenes del Bicentenario y al Programa de Doctorado en Ecología y Recursos Naturales de la Universidad Federal de São Carlos-Brasil.

\section{Referencias}

Alcaldía de Armenia. 2009. Plan de ordenamiento territorial de Armenia. [11 Enero 2013] URL:http://www.armenia.gov. co/index.php?controlador $=$ POT

Alcaldía de Buenaventura. 2001. Plan de ordenamiento territorial de Buenaventura. [11 Enero 2013]

URL:http://www.buenaventurasi.com/index. php? option=com_content $\&$ view $=$ article \&i $\mathrm{d}=59$ \& Itemid $=29$

Alcaldía de Neiva. 2000. Acuerdo 016 Plan de ordenamiento territorial de Neiva. [21 Marzo 2013]

URL:http://www.alcaldianeiva.gov.co/cms/in dex. php?option $=$ com_wrapper \&view $=$ wra pper\&Itemid $=141$

Alcaldía de Neiva. 2009. Acuerdo 026 Modifica el plan de ordenamiento territorial de Neiva. [21 Marzo 2013]

URL:http://aplicaciones.alcaldianeiva.gov.co /gaceta/repository/index/file/993

Alcaldía de Neiva. 2010. Decreto 596 Tratamiento urbanístico de Neiva. [11 Enero 2013]

URL:http://aplicaciones.alcaldianeiva.gov.co /gaceta/repository/index/file/1321

Alcaldía de Santa Marta (Magdalena, Colombia). 2000. Plan de ordenamiento territorial de Santa Marta. [21 Marzo 2013]

URL:http://www.invemar.org.co/archivo.jsp? id $=3417 \&$ red $=$ true

Alcaldía de Santa Marta (Magdalena, Colombia). 2012. Revisión y ajuste del POT de Santa Marta. [21 Marzo 2013]. 
URL:http://santamarta-magdalena. gov.co/apc-aa-files/3832653334363433 6335636234323637/PRESENTACION_RE VISION_POT_Modo_de_compatibilidad _.pdf

Alcaldía de Villavicencio. 2000. Decreto 353, por el cual se adopta el POT del municipio de Villavicencio. [22 Julio 2012]

URL:http://www.alcaldiadevillavicencio.gov. co/ws/documentos/Decreto $\% 20353 \% 20 d$ e\%202000\%20POT.pdf

Alcaldía de Villavicencio. 2009. Expediente municipal. [22 Mayo 2012]

URL:http://www.alcaldiadevillavicencio. gov $\cdot \mathrm{co} / \mathrm{ws} /$ ?categoria $=33 \&$ seccion $=201$

Alcaldía de Villavicencio. 2011. Proyecto de Acuerdo 018, por el cual se modifica el POT del municipio de Villavicencio. [15 Enero 2013]

URL:http://www.alcaldiadevillavicencio.gov. co/ws/Documentos/Pro-Acu-018-2011.pdf

Alcaldía de Villavicencio. 2012. Información general de la ciudad de Villavicencio. [17 Mayo 2012]

URL:http://www.alcaldiadevillavicencio.gov. $\mathrm{co} / \mathrm{ws} /$ ?categoria $=9 \&$ seccion $=41$

Armenteras D, González AF, Franco AC. Distribución espacial y temporal de incendios en Colombia utilizando anomalías térmicas. Caldasia. 2009; 31:291-306.

Armenteras-Pascual D, Retana-Alumbreros J, Molowny-Horas R. Characterizing fire spatial pattern interactions with climate and vegetation in Colombia. Agricultural and Forest Meterology. 2011; 151:279-289.
Baker S. 2006. Sustainable Development. 1st ed., Routledge, New York, p. 15.

Carrión GA. Debilidades del nivel regional en el ordenamiento territorial colombiano: Aproximación desde la normatividad política administrativa y de usos del suelo. Arquitectura, Ciudad y Entorno. 2008; 3(7):145-166.

Curaduría de Manizales. 2001. Acuerdo 508 Plan de Ordenamiento Territorial de Manizales. [11 Enero 2013]

URL:http://www.curaduriamanizales.com/do Cs/AcuerdoNo.508Oct.122001.pdf

Curaduría de Manizales. 2003. Acuerdo 573 Modifica el plan de ordenamiento territorial de Manizales. [11 Enero 2013]

URL:http://www.curaduriamanizales.com/do Cs/AcuerdoNo.573Dic.242003.pdf

Curaduría de Manizales. 2007. Acuerdo 663 Modifica el plan de ordenamiento territorial de Manizales. [11 Enero 2013]

URL:http://www.curaduriamanizales.com/do cs/AcuerdoNo663Sep132007.pdf

DANE - Departamento Administrativo Nacional de Estadística. 2013. Estimación y proyección de población nacional, departamental y municipal total por área 1985-2020. [21 Marzo 2013]

URL:http://www.dane.gov.co/index.php?opti on $=$ com_content $\&$ view $=$ article $\& i d=75 \& \mathrm{lt}$ emid $=72$

Escobar G. 2013. Historia de la legislación ambiental de Colombia. [22 Marzo 2013]

URL:http://es.calameo.com/read/000366553 5 fdcd838e765 
Espinosa MA. Ley 388 de 1997: una década de fortalecimiento legal del ordenamiento ilegal. Arquitectura, Ciudad y Entorno. 2008; 3(7):177-198.

Etter A, McAlpine C, Phinn S, Pullar D, Possingham $\mathrm{H}$. Unplanned land clearing of Colombian rainforests: Spreading like disease?. Landscape and urban planning 2006 ; 77 : 240-254.

Etter A, McAlpine C, Possingham H. Historical patterns and drivers of landscape change in Colombia since 1500: A regionalized Spatial Approach. Annals of the Association of American Geographers. 2008; 98(1): $2-23$.

García L. Teoría del desarrollo sostenible y legislación ambiental colombiana: una reflexión cultural. Universidad del Norte. Revista de Derecho. 2003; 20:198-215.

IGAC - Instituto Geográfico Agustín Codazzi. 2003. Mapa físico-político del Departamento del Meta. [17 Mayo 2012]

URL:http://www.vmapas.com/America/Colo mbia/Meta/Mapa_Fisico_Politico_Meta_C olombia_2003.jpg/maps-es.html

IDEAM - Instituto de Estudios Ambientales. 2004. Estadísticas. [10 Agosto 2012] URL: http://www.ideam.gov.co/publica/index4. htm

Gómez D, Salas A, Suarez C. 2007. Itinerario y énfasis del ordenamiento territorial en Colombia: Reflexiones al cumplir la Ley de Desarrollo Territorial, Ley 388 de 1997, una década de vigencia. [28 Marzo 2013] URL:http://repository.urosario.edu.co/bitst ream/10336/1216/1/BI\%2026CP.pdf

Gutiérrez F. Organización territorial, desarrollo sostenible y nuevas visiones sobre el territorio en Colombia (1991-
2010). Perspectiva geográfica. 2010; 15 : 239-260.

Massiris A. 2000. Ordenamiento territorial: experiencias internacionales y desarrollos conceptuales y legales realizados en Colombia. Biblioteca virtual Banco de la República. [18 Diciembre 2012]

URL:http://www.banrepcultural.org/blaavirtu al/geografia/orden/presen.htm

Massiris A. 2002. Ordenación del territorio en America Latina. Scripta Nova 6(125); [30 Enero 2013] URL: http://www.ub.edu/ geocrit/sn/sn-125.htm

Mendoza JE, Etter A. Multitemporal analysis (1940-1996) of land cover changes in the southwestern Bogotá highplain (Colombia). Landscape and urban planning 2002; 59:147-158.

Mittermeier RA, Gil PR, Mittermeier CG. 1997. Megadiversity. Earth's Biologically Wealthiest Nations. CEMEX, Mexico City. p.282-297.

Observatorio Legislativo. 2011. Ley Organica de Ordenamiento Territorial-LOOT (Ley 1454). Instituto de Ciencia Política, Boletin 186. [19 Diciembre 2012]

URL:http://www.icpcolombia.org/archivos/o bservatorio/boletin_186

Prieto JB, Luengas EC. 2011. La ley orgánica de ordenamiento territorial, como instrumento para la integración del ordenamiento territorial y ambiental. [27 Marzo 2013]

URL:http://www.umng.edu.co/documents/10 162/745281/V3N2_24.pdf

Rausch J. 2007. From frontier town to metropolis: A history of Villavicencio, 
Colombia, since 1842. Rowman \& Littlefield Publishers Inc, Plymouth. p.12231.

Rauschmayer F, Omann I, Frühmann J. 2012. Sustainable development: Capabilities, needs and well-being. Routledge studies in ecological economics. New York. p. 200216.

Rodríguez LE, Rodríguez AL, Borja JH, Ceballos DL, Uribe C, Murillo A, Arias R. 2011. Historia de Colombia, todo lo que hay que saber. Grupo Santillana S.A. Bogotá. p.15-366.
Ucros JC. 2008. Breve historia y situación actual del patrimonio forestal colombiano. [28 Junio 2012]

URL:http://www.fao.org/forestry/1727209 c 7 b b 88 c ba ad 85 cf 5 c 312 d 8422 b30afb.pdf

Yory CM. Desarrollo territorial integrado, ciudad difusa y nuevas ruralidades: Consideraciones propositivas para fortalecer el tema de la ciudad-región en el diseño y revisión de los POT. Acta Geográfica. 2007; 1(1):11-28. 